\title{
Holomorphic functions of fast growth on submanifolds of the domain
}

\author{
by Piotr JakóBCZaK (Kraków)
}

\begin{abstract}
We construct a function $f$ holomorphic in a balanced domain $D$ in $\mathbb{C}^{N}$ such that for every positive-dimensional subspace $\Pi$ of $\mathbb{C}^{N}$, and for every $p$ with $1 \leq p<\infty$, $\left.f\right|_{\Pi \cap D}$ is not $L^{p}$-integrable on $\Pi \cap D$.
\end{abstract}

1. Introduction. Let $D$ be an open set in $\mathbb{C}^{N}$, and let $F$ be some class of complex-valued functions in $D$ which are holomorphic in $D$ and satisfy some other conditions there. Given an affine subspace $M$ of positive dimension in $\mathbb{C}^{N}$, the problem is to determine what further properties (besides being holomorphic) the functions from the class $F$ have when restricted to the slice $M \cap D$. This problem was studied in many situations by several authors; see e.g. [2], [5], [8], [9], [11].

In [4] we have shown that there exists a function $f$ holomorphic in the unit ball $B$ in $\mathbb{C}^{N}$ such that for every positive-dimensional subspace $\Pi$ of $\mathbb{C}^{N},\left.f\right|_{\Pi \cap B}$ is not $L^{2}$-integrable in $\Pi \cap B$. The proof consists of construction of a function $f$ with sufficiently fast growth near the boundary of each set of the form $\Pi \cap B$, and the use of the well-known estimates relating the growth near the boundary and the $L^{2}$-norm of a holomorphic function. (See also [10] for a much more explicit proof of this result.)

In the present note we carry out the construction from [4] for the more general situation of domains which are balanced domains of holomorphy, i.e. domains of holomorphy such that for every $z=\left(z_{1}, \ldots, z_{N}\right) \in D$ and every $\lambda \in \mathbb{C}$ with $|\lambda| \leq 1$, the point $\lambda z=\left(\lambda z_{1}, \ldots, \lambda z_{N}\right)$ also belongs to $D$. We obtain holomorphic functions with prescribed fast growth near the boundary of such domains; then we apply our construction in order to obtain functions which are holomorphic and not integrable on linear slices of the domain, or which are not in $\mathcal{O}(\delta)$ on any such slice, where $\mathcal{O}(\delta)$ denotes the

1991 Mathematics Subject Classification: 32A07, 32A37.

Key words and phrases: balanced domains, growth of holomorphic function.

Partially supported by the KBN Grant 2 PO3A 06008. 
space of functions of $\delta$-tempered growth, and $\delta$ is a given weight function (see e.g. [1]; the precise definiton of $\mathcal{O}(\delta)$ will be recalled later).

The author is very indebted to M. Jarnicki, J. Siciak, and P. Wojtaszczyk for valuable suggestions and discussions.

2. A holomorphic function with prescribed growth on slices. Let $D$ be a balanced domain of holomorphy in $\mathbb{C}^{N}$. Then there exists a strictly plurisubharmonic smooth exhaustion function $\varrho$ in $D$, i.e. a smooth function $\varrho$ which is strictly plurisubharmonic in $D$ and for every real $c$, the set $\{z \in D \mid \varrho(z)<c\}$ is relatively compact in $D$. For further use we need the existence of a sequence $\left\{D_{n}\right\}_{n=1}^{\infty}$ of strictly pseudoconvex, smoothly bounded, balanced domains which exhaust $D$ and every straight line in $\mathbb{C}^{N}$ passing through zero intersects the boundary $\partial D_{n}$ of every domain $D_{n}$ transversally. It seems that the existence of such a sequence is well known; the proof of the following proposition was suggested to us by M. Jarnicki, Ch. Kiselman and P. Pflug.

Proposition 1. Let $D$ be a balanced domain of holomorphy in $\mathbb{C}^{N}$. Then there exists $\varepsilon_{0}>0$ and a family $\left\{D_{\varepsilon}\right\}_{0<\varepsilon<\varepsilon_{0}}$ of strictly pseudoconvex, smoothly bounded, balanced domains such that $\bigcup_{0<\varepsilon \leq \varepsilon_{0}} D_{\varepsilon}=D, \bar{D}_{\varepsilon} \subset D_{\varepsilon^{\prime}}$ for $0<\varepsilon^{\prime}<\varepsilon \leq \varepsilon_{0}$, and for every $\varepsilon$, every (real) straight line passing through zero in $\mathbb{C}^{N}$ intersects $\partial D_{\varepsilon}$ transversally.

Proof. Let $h$ be the Minkowski functional for $D$. Since $D$ is a domain of holomorphy and is balanced, $h$ is plurisubharmonic in $\mathbb{C}^{N}$, and $h(\lambda z)=|\lambda| h(z)$ for every $z \in \mathbb{C}^{N}$ and $\lambda \in \mathbb{C}$. For $\varepsilon>0$, denote by $h_{\varepsilon}$ the regularization

$$
h_{\varepsilon}(z)=\int_{\mathbb{C}^{N}} h(z-\varepsilon y) \phi(y) d m(y),
$$

where $\phi$ is a smooth function in $\mathbb{C}^{N}, \operatorname{supp} \phi$ is the unit ball, $\phi(y)=\phi\left(\left|y_{1}\right|, \ldots\right.$ $\left.\ldots,\left|y_{N}\right|\right)$ for every $y=\left(y_{1}, \ldots, y_{N}\right) \in \mathbb{C}^{N}$, and $\int_{\mathbb{C}^{N}} \phi(y) d m(y)=1$. (Here $m$ denotes the usual Lebesgue measure in $\mathbb{C}^{N}$.) It is well known that $h_{\varepsilon}$ is smooth and plurisubharmonic in $\mathbb{C}^{N}$, for each $z \in \mathbb{C}^{N}, h_{\varepsilon}(z)$ tends decreasingly to $h(z)$ as $\varepsilon$ decreases to zero, and $h_{\varepsilon}\left(e^{i t} z\right)=h_{\varepsilon}(z), z \in \mathbb{C}^{N}, t \in \mathbb{R}$. Since $h(0)=0$, there exists $\varepsilon_{0}>0$ so small that $h_{\varepsilon_{0}}(0)<1$ (and hence $h_{\varepsilon}(0)<1$ for all $\left.0<\varepsilon \leq \varepsilon_{0}\right)$. For $0<\varepsilon \leq \varepsilon_{0}$, set

$$
\varrho_{\varepsilon}(z)=h_{\varepsilon}(z)+\varepsilon\|z\|^{2} .
$$

Then $\varrho_{\varepsilon}$ is a smooth and strictly plurisubharmonic function in $\mathbb{C}^{N}$. Let $D_{\varepsilon}=$ $\left\{z \in \mathbb{C}^{N} \mid \varrho_{\varepsilon}(z)<1\right\}$. Then $0 \notin \partial D_{\varepsilon}$ (because $\left.\varrho_{\varepsilon}(0)<1\right), \bar{D}_{\varepsilon^{\prime}} \subset D_{\varepsilon^{\prime \prime}}$ for $0<\varepsilon^{\prime \prime}<\varepsilon^{\prime}$, and the domains $D_{\varepsilon}$ tend increasingly to $D$ as $\varepsilon$ decreases to zero; moreover, every domain $D_{\varepsilon}$ is pseudoconvex. Using the maximum 
principle for subharmonic functions and the fact that $h_{\varepsilon}\left(e^{i t} z\right)=h_{\varepsilon}(z)$ for $z \in \mathbb{C}^{N}$ and $t \in \mathbb{R}$, we have for $\lambda \in \mathbb{C},|\lambda| \leq 1$ and $z \in \mathbb{C}^{N}$,

$$
h_{\varepsilon}(\lambda z) \leq \max _{t \in \mathbb{R}} h_{\varepsilon}\left(e^{i t} z\right)=h_{\varepsilon}(z)
$$

and hence

$$
\varrho_{\varepsilon}(\lambda z) \leq \varrho_{\varepsilon}(z), \quad z \in \mathbb{C}^{N}, \lambda \in \mathbb{C},|\lambda| \leq 1
$$

Hence every domain $D_{\varepsilon}$ is balanced.

Now fix $\varepsilon$ with $0<\varepsilon \leq \varepsilon_{0}$, and $z \in \partial D_{\varepsilon}$. By (1) the function

$$
\phi:[0, \infty) \ni t \mapsto h_{\varepsilon}(t z)
$$

is non-decreasing. Denote by $\psi$ the function

$$
\psi:[0, \infty) \ni t \mapsto \varrho_{\varepsilon}(t z) .
$$

Then

$$
\psi^{\prime}(t)=\left\langle\operatorname{grad} \varrho_{\varepsilon}(t z), z\right\rangle_{\mathbb{R}}=\phi^{\prime}(t)+2 \varepsilon t\|z\|^{2},
$$

where $\langle\cdot, \cdot\rangle_{\mathbb{R}}$ denotes the standard real scalar product in $\mathbb{C}^{N}=\mathbb{R}^{2 N}$. Further,

$$
\left\langle\operatorname{grad} \varrho_{\varepsilon}(z), z\right\rangle_{\mathbb{R}}=\phi^{\prime}(1)+2 \varepsilon\|z\|^{2}>0
$$

(here we use the fact that $0 \notin \partial D_{\varepsilon}$ ). It follows form (2) that $\partial D_{\varepsilon}$ is smooth (and so $D_{\varepsilon}$ is strictly pseudoconvex), and that

(3) $\partial D_{\varepsilon}$ is transversal to every (real) straight line passing through zero.

This ends the proof.

Fix $\varepsilon$ with $0<\varepsilon \leq \varepsilon_{0}$. It is well known that for a given compact subset $K$ of $\mathbb{C}^{N}$, and for $\varepsilon^{\prime}$ sufficiently close to $\varepsilon$, the regularizations $h_{\varepsilon^{\prime}}$ are arbitrarily close to $h_{\varepsilon}$ on $K$. Therefore the same is true for the functions $\varrho_{\varepsilon^{\prime}}$ and $\varrho_{\varepsilon}$. Hence, given an arbitrary neighborhood $U$ of $\bar{D}_{\varepsilon}$, there exists $\varepsilon^{\prime}<\varepsilon$ such that $D_{\varepsilon^{\prime}} \subset U$. Suppose now that $f$ is a function holomorphic in some neighborhood $U$ of $\bar{D}_{\varepsilon}$, and fix $D_{\varepsilon^{\prime}} \subset U$ as above. Then $f$ is holomorphic in $D_{\varepsilon^{\prime}}$. Since $D_{\varepsilon^{\prime}}$ is a balanced domain of holomorphy, there exists a series $\sum_{s=0}^{\infty} Q_{s}$ of homogeneous polynomials which converges to $f$ uniformly on compact subsets of $D_{\varepsilon^{\prime}}$; in particular, the convergence is uniform on $\bar{D}_{\varepsilon}$. This yields the following proposition:

Proposition 2. Let the domain $D$ and the family $\left\{D_{\varepsilon}\right\}_{0<\varepsilon \leq \varepsilon_{0}}$ be as in Proposition 1. Then given $\varepsilon$ with $0<\varepsilon \leq \varepsilon_{0}$, every function holomorphic in a neighborhood of $\bar{D}_{\varepsilon}$ can be uniformly approximated on $\bar{D}_{\varepsilon}$ by functions which are holomorphic in the whole domain $D$.

In the sequel, given $K \subset \mathbb{C}^{N}$ and $f \in \mathcal{C}(K)$, we denote by $\|f\|_{K}$ the usual supremum norm on $K$.

Suppose now that $\delta$ is a positive, bounded and continuous function in a domain $G$ in $\mathbb{C}^{N}$. Denote by $\mathcal{O}(\delta)$ the space of all functions holomorphic in 
$G$ such that there exists a positive integer $k$ with

$$
\sup \left\{\left|\delta^{k}(z) f(z)\right| \mid z \in G\right\}<\infty .
$$

If moreover $\delta$ satisfies the conditions:

(i) $|z| \delta$ is bounded on $\mathbb{C}^{N}$,

(ii) $\left|\delta(z)-\delta\left(z^{\prime}\right)\right| \leq\left|z-z^{\prime}\right|$ for all $z, z^{\prime} \in \mathbb{C}^{N}$,

then it is called a weight function (see [1]). The theory of functions from the space $\mathcal{O}(\delta)$ was investigated by several authors (see e.g. [1]).

We will prove the following theorem on the existence of holomorphic functions with bad boundary behavior on submanifolds:

TheOREM 1. Let $D$ be a balanced domain of holomorphy in $\mathbb{C}^{N}$, and $\delta$ a positive and continuous function in $D$. Then there exists a function $f$ holomorphic in $D$ such that for every positive-dimensional subspace $\Pi$ of $\mathbb{C}^{N},\left.f\right|_{\Pi \cap D} \notin \mathcal{O}\left(\left.\delta\right|_{\Pi \cap D}\right)$.

Let $\left\{D_{\varepsilon}\right\}_{0<\varepsilon \leq \varepsilon_{0}}$ be the family of domains constructed in Proposition 1 . Choose an arbitrary sequence $\left\{\varepsilon_{n}\right\}_{n=1}^{\infty}$ with $\varepsilon_{0}>\varepsilon_{1}>\varepsilon_{2}>\ldots$ and $\lim _{n \rightarrow \infty} \varepsilon_{n}=0$. We have $\bar{D}_{\varepsilon_{n}} \subset D_{\varepsilon_{n+1}}$ for $n=1,2, \ldots$ For each $n$, choose a neighborhood $U_{n}$ of $\partial D_{\varepsilon_{n}}$ such that $\bar{U}_{n} \subset D$, and $\bar{U}_{n} \cap \bar{U}_{k}=\emptyset$ for $n \neq k$. It follows from the proof of Proposition 1 that for every $n$ and every $z \in \partial D_{\varepsilon_{n}}$, $\operatorname{grad} \varrho_{\varepsilon_{n}}(z) \neq 0$ (where $\varrho_{\varepsilon_{n}}$ is a defining function for $D_{\varepsilon_{n}}$, obtained in the proof of Proposition 1). Shrinking the neighborhoods $U_{n}$ if necessary we may assume that

$$
\text { for every } n \text { and for every } z \in U_{n}, \quad \operatorname{grad} \varrho_{\varepsilon_{n}}(z) \neq 0 .
$$

Moreover, according to the proof of Proposition 1, we have

$$
D_{\varepsilon_{n}}=\left\{z \in \mathbb{C}^{N} \mid \varrho_{\varepsilon_{n}}(z)<1\right\},
$$

and $\varrho_{\varepsilon_{n}}$ is smooth and strictly plurisubharmonic in $\mathbb{C}^{N}$, and satisfies the condition

$$
\varrho_{\varepsilon_{n}}(\lambda z) \leq \varrho_{\varepsilon_{n}}(z), \quad z \in \mathbb{C}^{N}, \lambda \in \mathbb{C},|\lambda| \leq 1 .
$$

Therefore there exists a positive number $\omega_{n}$ such that for every $0<\omega \leq \omega_{n}$, the domains

$$
D_{\varepsilon_{n},-\omega}=\left\{z \in \mathbb{C}^{N} \mid \varrho_{\varepsilon_{n}}(z)<1-\omega\right\}
$$

are strictly pseudoconvex, smoothly bounded, and balanced, $\overline{D_{\varepsilon_{n}} \backslash U_{n}} \subset$ $D_{\varepsilon_{n},-\omega}$, and (as in (3)) $\partial D_{\varepsilon_{n},-\omega}$ is transversal to every (real) straight line passing through zero.

Now fix $n \in \mathbb{N}$, and call $D_{\varepsilon_{n}}=G, \varrho_{\varepsilon_{n}}=\varrho, D_{\varepsilon_{n},-\omega}=G_{-\omega}, U_{n}=U$. It is well known that every strictly pseudoconvex domain is locally strictly convex with respect to convenient holomorphic coordinates in some neighborhood of a given point of its boundary. Examining the proof of this result 
(see e.g. [6], Lemma 3.2.3), and shrinking $U$ once more, we conclude that the following holds:

Proposition 3. For every $x \in \partial G$ there exist neighborhoods $Z_{x}, U_{x}, V_{x}$, and $W_{x}$ of $x$ with $Z_{x} \Subset U_{x} \Subset V_{x} \Subset W_{x}$, strictly convex domains $P_{x}, T_{x}, S_{x}$, and $R_{x}$ in $\mathbb{C}^{N}$ such that $P_{x} \Subset T_{x} \Subset S_{x} \Subset R_{x}$, and a biholomorphic mapping $\phi_{x}: W_{x} \rightarrow R_{x}$ such that

$$
\varrho_{n} \circ \phi_{x}^{-1} \text { is a strictly convex smooth function in } R_{x} \text {, }
$$

$\phi_{x}\left(Z_{x}\right)=P_{x}, \phi_{x}\left(U_{x}\right)=T_{x}, \phi_{x}\left(V_{x}\right)=S_{x}$, and

$$
\bar{U} \subset \bigcup_{x \in \partial G} Z_{x} .
$$

Now let $x \in \partial G$ be fixed. By a small perturbation of the function $\varrho_{n}$ we can obtain a strictly pseudoconvex domain $B \subset \mathbb{C}^{N}$ with smooth boundary such that $B \subset G, G \cap U_{x} \subset B,\left(\partial G \backslash V_{x}\right) \cap \bar{B}=\emptyset, \phi_{x}\left(B \cap W_{x}\right)$ is convex, there exists $\eta$ with $0<\eta<\omega_{n}$ such that $\bar{G}_{-\eta} \subset B$, and $B$ is star-shaped. (Note that since the deformation of $G$ is performed only near $x \in \partial G$, the domain $B$ need not be balanced (although $G$ is). Therefore $B$ is a star-shaped domain of holomorphy. It follows from [9] that every function holomorphic in $B$ can be approximated uniformly on compact subsets of $B$ by polynomials. In particular,

(10) every function holomorphic in $B$ can be approximated uniformly on compact subsets of $B$ by functions holomorphic in the whole domain $D$.

Also, there exists $\theta$ with $0<\theta<\eta$ such that $\left(\left(\overline{G \backslash G_{-\theta}}\right) \backslash V_{x}\right) \cap B=\emptyset$, and hence

$$
B \cap\left(W_{x} \backslash V_{x}\right) \subset W_{x} \cap G_{-\theta} .
$$

Assume now that $K$ and $L$ are compact subsets of $\phi_{x}\left(\left(G \backslash G_{-\theta}\right) \cap U_{x}\right)$ such that

$K$ is a subset of a real $(2 N-1)$-dimensional hyperplane $\Pi$ of $\mathbb{C}^{N}$, and $\phi_{x}\left(G_{-\theta} \cap W_{x}\right)$ and $L$ lie on one side of $\Pi$.

(This can happen, since by (8), $\phi_{x}\left(G_{-\theta} \cap W_{x}\right)$ is convex in $\mathbb{C}^{N}$.) The hyperplane $\Pi$ has the form

$$
\Pi=\left\{z \in \mathbb{C}^{N} \mid \operatorname{Re}\left\langle z-c z_{0}, z_{0}\right\rangle_{\mathbb{C}}=0\right\}
$$

with some $z_{0} \in \mathbb{C}^{N},\left\|z_{0}\right\|=1$, and $c>0$. (Here $\langle\cdot, \cdot\rangle_{\mathbb{C}}$ denotes the standard complex euclidean scalar product in $\mathbb{C}^{N}$.) The function

$$
h(z)=b \exp \left(a\left\langle z-c z_{0}, z_{0}\right\rangle_{\mathbb{C}}\right), \quad z \in \mathbb{C}^{N}, a, b>0,
$$

is such that $|h|_{\Pi} \mid \equiv b$, and $|h(z)|<b$ for those $z \in \mathbb{C}^{N}$ which lie on the same side of the hyperplane $\Pi$ as the point 0 . 
Choosing conveniently $a$ and $b$, and using (12), we may assume that

$$
\|h\|_{\phi_{x}\left(G_{-\theta} \cap W_{x}\right) \cup L} \leq m^{\prime},
$$

and

$$
\inf _{K}|h| \geq M^{\prime}
$$

where $0<m^{\prime}<M^{\prime}$ are given constants. Let $\psi$ be a smooth function in $\mathbb{C}^{N}$ with $0 \leq \psi \leq 1,\left.\psi\right|_{V_{x}} \equiv 1$, and $\left.\psi\right|_{\mathbb{C}^{N} \backslash W_{x}} \equiv 0$. Consider the function $g$ defined as $\psi\left(h \circ \phi_{x}\right)$ in $W_{x}$ and 0 in $\mathbb{C}^{N} \backslash W_{x}$. Then $g$ is smooth in $\mathbb{C}^{N}$. The form $\bar{\partial} g$ is $\bar{\partial}$-closed in $\mathbb{C}^{N}$, and

$$
\operatorname{supp} \bar{\partial} g \subset W_{x} \backslash V_{x} .
$$

Moreover, by (11), (13), and (15),

$$
\begin{aligned}
\|\bar{\partial} g\|_{\bar{B}} & =\left\|\left(h \circ \phi_{n, x}\right) \bar{\partial} \psi\right\|_{\bar{B}} \leq\|h\|_{\phi_{x}\left(B \cap\left(W_{x} \backslash V_{x}\right)\right)}\|\bar{\partial} \psi\|_{\mathbb{C}^{N}} \\
& \leq\|h\|_{\phi_{x}\left(W_{x} \cap G_{-\theta}\right)}\|\bar{\partial} \psi\|_{\mathbb{C}^{N}} \leq m^{\prime}\|\bar{\partial} \psi\|_{\mathbb{C}^{N}} .
\end{aligned}
$$

By [3] or [7] there exists $c>0$ (depending only on $B$ ) and a function $v \in \mathcal{C}^{\infty}(\bar{B})$ such that $\bar{\partial} v=\bar{\partial} g$ in $\bar{B}$, and

$$
\|v\|_{\bar{B}} \leq c m^{\prime}\|\bar{\partial} \psi\|_{\mathbb{C}^{N}}
$$

Then $f=g-v$ is holomorphic in $B$, and

$$
\|f\|_{G_{-\eta} \cup \phi_{x}^{-1}(L)} \leq\|h\|_{\phi_{x}\left(G_{-\theta} \cap W_{x}\right) \cup L}+\|v\|_{\bar{B}} \leq m^{\prime}+c m^{\prime}\|\bar{\partial} \psi\|_{\mathbb{C}^{N}},
$$

and by (14),

$$
\inf _{\phi_{x}^{-1}(K)}|f| \geq \inf _{K}|h|-\|v\|_{B} \geq M^{\prime}-c m^{\prime}\|\bar{\partial} \psi\|_{\mathbb{C}^{N}} .
$$

Hence, by choosing $M^{\prime}$ and $m^{\prime}$ conveniently, we obtain

$$
\inf _{\phi_{x}^{-1}(K)}|f| \geq M
$$

and

$$
\|f\|_{G_{-\eta} \cup \phi_{x}^{-1}(L)}<m
$$

where $0<m<M$ are given positive numbers.

By (10) there exists a function $k$ holomorphic in $D$ such that

$$
\inf \left\{|k(z)| \mid z \in \phi_{x}^{-1}(K)\right\}>M
$$

and

$$
\|k\|_{G_{-\eta} \cup \phi_{x}^{-1}(L)}<m .
$$

We now return to the previous notations, i.e. we have the sequence $\left\{D_{\varepsilon_{n}}\right\}_{n=1}^{\infty}$ of balanced, strictly pseudoconvex, and smoothly bounded domains from (5), defined by the smooth and strictly plurisubharmonic functions $\varrho_{\varepsilon_{n}}$ satisfying (6), and the numbers $\omega_{n}$ for which (7) holds. To simplify 
notations, we write $D_{\varepsilon_{n}}=D_{n}$ and $D_{\varepsilon_{n},-\omega}=D_{n,-\omega}$. Let $n$ be fixed. Since $\bar{U}_{n}$ is compact, by (9) there exist a finite number of points $x_{n, 1}, \ldots, x_{n, i_{n}} \in \partial D_{n}$ such that $\bar{U}_{n} \subset Z_{n, x_{n, 1}} \cup \ldots \cup Z_{n, x_{n, i_{n}}}$. Let $S$ be the unit sphere in $\mathbb{C}^{N}$, $S=\left\{w \in \mathbb{C}^{N} \mid\|w\|=1\right\}$. Note that for every $w \in S$, the half-line $I_{w}=\{t w \mid 0 \leq t<\infty\}$ intersects every $\partial D_{n,-\omega}, 0<\omega \leq \omega_{n}$. Hence

$$
\text { every } I_{w} \text { intersects some } Z_{n, x_{n, j}} \text {. }
$$

Moreover, by Proposition 3, every such half-line $I_{w}$ intersects every $\partial D_{n,-\omega}$, $0<\omega \leq \omega_{n}$, transversally. By (8), for every $j=1, \ldots, i_{n}$, the sets

$$
\phi_{n, x_{n, j}}\left(D_{n,-\omega} \cap W_{n, x_{n, j}}\right)
$$

are convex in $\mathbb{C}^{N}$ for every $0<\omega \leq \omega_{n}$, and the lines $\phi_{n, x_{n, j}}\left(I_{w}\right)$ intersect $\phi_{n, x_{n, j}}\left(\partial D_{n,-\omega} \cap W_{n, x_{n, j}}\right)$ transversally (for those $w$ and $\omega$ for which the intersection is not empty). Hence it is rather easy to find for each $j=1, \ldots, i_{n}$ a finite number of real $(2 N-1)$-dimensional hyperplanes $\Theta_{n, j, 1}, \ldots, \Theta_{n, j, s_{n, j}}$ of $\mathbb{C}^{N}$, a family $K_{n, j, 1}, \ldots, K_{n, j, s_{n, j}}$ of compact subsets of $\mathbb{C}^{N}$, and a number $\omega_{n, j}$ with $0<\omega_{n, j}<\omega_{n}$, as well as a number $\omega_{n, 0}, 0<\omega_{n, 0}<\omega_{n}$, such that:

- $K_{n, j, l} \subset \Theta_{n, j, l} \cap T_{n, x_{n, j}}, \quad l=1, \ldots, s_{n, j}$.

(21) If for some $w \in S$, the half-line $I_{w}$ intersects $Z_{n, x_{n, j}}$, then $\phi_{n, x_{n, j}}\left(I_{w}\right)$ (which is contained in $W_{n, x_{n, j}}$ ) intersects some $K_{n, j, l}$.

- For every $l=1, \ldots, s_{n, j}$, the sets $\overline{\phi_{n, x_{n, j}}\left(W_{n, x_{n, j}} \cap D_{n,-\omega_{n, j}}\right)}$ and $K_{n, j, 1}, \ldots, K_{n, j, l-1}$ lie on the same side of $\Theta_{n, j, l}$ as the point zero,

(we set $K_{n, j, 0}=\emptyset$ ),

$$
\omega_{n, 0}>\omega_{n, 1}>\ldots>\omega_{n, i_{n}}, \quad \text { so } \quad D_{n,-\omega_{n, 1}} \Subset \ldots \Subset D_{n,-\omega_{n, i_{n}}},
$$

and

$$
\begin{aligned}
K_{n, j, l} \subset \phi_{n, x_{n, j}}\left(W_{n, x_{n, j}} \cap\left(D_{n,-\omega_{n, j}} \backslash \bar{D}_{n,-\omega_{n, j-1}}\right)\right), \\
\\
\quad j=1, \ldots, i_{n}, l=1, \ldots, s_{n, j} .
\end{aligned}
$$

Now we repeat essentially the construction from [4]. We order the sets $K_{n, j, l}$ into the sequence

$$
\begin{aligned}
& \left\{K_{1,1,1}, K_{1,1,2}, \ldots, K_{1,1, s_{1,1}}, K_{1,2,1}, \ldots, K_{1,2, s_{1,2}}, \ldots\right. \\
& \left.\quad K_{1, i_{1}, 1}, \ldots, K_{1, i_{1}, s_{1, i_{1}}}, K_{2,1,1}, \ldots, K_{2,1, s_{2,1}}, \ldots\right\}=:\left\{K_{1}, K_{2}, \ldots\right\} .
\end{aligned}
$$

Every subspace $\Pi$ of $\mathbb{C}^{N}$ consists of real half-lines $I_{w}$, and, by (20) and (21),

for every $w \in S$, the half-line $I_{w}$ intersects infinitely many sets of the form $\phi_{n, x_{n, j}}^{-1}\left(K_{n, j, l}\right)$.

To each $K_{n, j, l}=K_{s}$ we attach a function $f_{n, j, l}=f_{s}$ with the properties which we now describe inductively. By (16) and (17), and by the positivity 
of $\delta$, there exists a function $f_{1}$ holomorphic in $D$ such that

$$
\inf \left\{\left|f_{1}(z)\right| \mid z \in \phi_{1, x_{1,1}}^{-1}\left(K_{1}\right)\right\} \geq 1 \quad \text { and } \quad\left\|\delta f_{1}\right\|_{\bar{D}_{1,-\omega_{1,1}}} \leq 2^{-1} .
$$

Suppose that the functions $f_{1}, \ldots, f_{r}$ are already chosen. Then we have

$$
K_{r+1}=K_{n_{r+1}, j_{r+1}, l_{r+1}}
$$

for uniquely determined $n_{r+1}, j_{r+1}$ with $1 \leq j_{r+1} \leq i_{n_{r+1}}$, and $l_{r+1}$ with $1 \leq l_{r+1} \leq s_{n_{r+1}, j_{r+1}}$. Moreover,

$$
\bar{D}_{n_{r+1},-\omega_{n_{r+1}, j}} \subset D_{n_{r+1},-\omega_{n_{r+1}, j_{r+1}}}, \quad j=1, \ldots, j_{r+1}-1, \quad \text { if } j_{r+1}>1,
$$

or

and the set

$$
\bar{D}_{n_{r},-\omega_{n_{r}, i_{n}}} \subset D_{n_{r+1},-\omega_{n_{r+1}, 1}} \quad \text { if } j_{r+1}=1,
$$

$$
\begin{aligned}
\phi_{n_{r+1}, x_{n_{r+1}, j_{r+1}}}\left(D_{n_{r+1},-\varepsilon_{n_{r+1}, j_{r+1}}} \cap W_{n_{r+1}, j_{r+1}}\right) \\
\cup K_{n_{r+1}, j_{r+1}, 1} \cup \ldots \cup K_{n_{r+1}, j_{r+1}, l_{r+1}-1}
\end{aligned}
$$

lies on the same side of the hyperplane $\Theta_{n_{r+1}, j_{r+1}, l_{r+1}}$ as the point zero. By (17)-(19) and the fact that $\delta$ is positive, there exists a function $f_{r+1}=$ $f_{n_{r+1}, j_{r+1}, l_{r+1}+1}$, holomorphic in $D$, such that

$$
\begin{aligned}
\inf \left\{\left|\delta^{r+1} f_{r+1}(z)\right| \mid z \in \phi_{n_{r+1}, x_{n_{r+1}, j_{r+1}}}^{-1}\left(K_{r+1}\right)\right\} & \\
& \geq(r+1)+\sum_{p=1}^{r}\left\|\delta^{r+1} f_{p}\right\|_{K_{r+1}}+1,
\end{aligned}
$$

and if we define

$$
\begin{aligned}
L_{r}= & D_{n_{r+1},-\omega_{n_{r+1}, j_{r+1}}} \\
& \cup \phi_{n_{r+1}, x_{n_{r+1}, j_{r+1}}}^{-1}\left(K_{n_{r+1}, j_{r+1}, 1} \cup \ldots \cup K_{n_{r+1}, j_{r+1}, l_{r+1}-1}\right),
\end{aligned}
$$

then

$$
\begin{gathered}
\left\|f_{r+1}\right\|_{L_{r}}\left(=\left\|f_{n_{r+1}, j_{r+1}, l_{r+1}+1}\right\|_{L_{r}}\right) \leq 2^{-(r+1)}, \\
\left\|\delta^{p} f_{r+1}\right\|_{L_{r}}\left(=\left\|\delta^{p} f_{n_{r+1}, j_{r+1}, l_{r+1}+1}\right\|_{L_{r}}\right) \leq 2^{-(r+1)}, \quad p=1, \ldots, r .
\end{gathered}
$$

Set

$$
f(z)=\sum_{r=1}^{\infty} f_{r}(z), \quad z \in D .
$$

By (25), the function $f$ is well defined and holomorphic in $D$. By (20), (23), (24), and (26), for every $w \in S$ there exists a sequence $\left\{z_{r}\right\}_{r=1}^{\infty}$ of points of $I_{w} \cap D$ such that for infinitely many $r$,

$$
\left|\delta^{r}\left(z_{r}\right) f\left(z_{r}\right)\right| \geq r .
$$

Therefore $f$ is not in $\mathcal{O}\left(\left.\delta\right|_{\Pi \cap D}\right)$ for any subspace $\Pi$ of $\mathbb{C}^{N}$. This ends the proof of Theorem 1. 
Given a domain $G$ in $\mathbb{C}^{N}$ and a number $p$ with $1 \leq p<\infty$, we denote by $L^{p} H(G)$ the space of all functions holomorphic in $G$ such that

$$
\int_{G}|f(z)|^{p} d m(z)<\infty
$$

( $m$ denotes here the $2 N$-dimensional Lebesgue measure in $\mathbb{C}^{N}$ ). If $G$ is a domain in a complex subspace $M$ of $\mathbb{C}^{N}$, the space $L^{p} H(G)$ can be defined similarly, with $m$ being the Lebesgue measure on $M$.

In the same way as Theorem 1 we can prove the following theorem on functions from the space $L^{p} H$ (for the case of the ball, see [4], Theorem 1):

Theorem 2. Let $D$ be a balanced domain of holomorphy in $\mathbb{C}^{N}$. Then there exists a function $f$, holomorphic in $D$, such that for every positivedimensional subspace $\Pi$ of $\mathbb{C}^{N}$ and for every $p$ with $1 \leq p<\infty,\left.f\right|_{D \cap \Pi} \notin$ $L^{p} H(D \cap \Pi)$.

Proof. It is well known that if $G$ is a domain in $\mathbb{C}^{M}, 1 \leq p<\infty$, and $f \in L^{p} H(G)$ then for every $z_{0} \in G$,

$$
\left|f\left(z_{0}\right)\right| \leq \frac{M^{M / p}}{\left(\pi \operatorname{dist}\left(z_{0}, \partial G\right)^{2}\right)^{M / p}}\|f\|_{G, p},
$$

where $\|f\|_{G, p}$ denotes the $L^{p}$-norm of $f$ in $G$ and $\operatorname{dist}\left(z_{0}, \partial G\right)$ is the Euclidean distance of $z_{0}$ to $\partial G$. For $z_{0}$ sufficiently close to $\partial G$, we have $\operatorname{dist}\left(z_{0}, \partial G\right)<1$. Hence for $1 \leq p<\infty$,

$$
1 \leq \frac{1}{\operatorname{dist}\left(z_{0}, \partial G\right)^{2 M / p}} \leq \frac{1}{\operatorname{dist}\left(z_{0}, \partial G\right)^{2 M}} .
$$

Therefore, for all $z_{0} \in G$, and for every $1 \leq p<\infty$, we have

$$
\frac{1}{\operatorname{dist}\left(z_{0}, \partial G\right)^{2 M / p}} \leq 1+\frac{1}{\operatorname{dist}\left(z_{0}, \partial G\right)^{2 M}} .
$$

Moreover, there exists $c>0$ such that for all $L=1, \ldots, N$, and every $1 \leq p<\infty$,

$$
(L / \pi)^{L / p} \leq c .
$$

Consider the construction of the function $f$ from the proof of Theorem 1 . We now require that the function $f$, constructed as before, satisfies the inequality

$$
|f(z)| \geq \frac{r}{\operatorname{dist}\left(z_{0}, \partial D\right)^{2 N}}+1
$$

for all $z \in K_{r}$ instead of (27). (Here the sets $K_{r}$ are defined as in (22)). It follows from the above considerations and from (28) that the function $f$ obtained in this way is holomorphic in $D$, and for every subspace $\Pi$ of $\mathbb{C}^{N}$ and every $1 \leq p<\infty, f \notin L^{p}(\Pi \cap D)$. This ends the proof. 
Now let $D$ be a balanced domain of holomorphy in $\mathbb{C}^{N}$, as before. Then in particular Theorem 2 holds for $D$ and $p=2$. Moreover, since $D$ is balanced, every function $f$ holomorphic in $D$ can be developed into a series of homogeneous polynomials,

$$
f(z)=\sum_{s=0}^{\infty} Q_{s}(z)
$$

where every $Q_{s}$ is a homogeneous polynomial of degree $s, s=0,1, \ldots$ In [10], Thm. 1, Wojtaszczyk constructed explicitly a sequence $\left\{p_{n}\right\}_{n=1}^{\infty}$ of homogeneous polynomials of degree $n$ in the unit ball $B$ in $\mathbb{C}^{N}$ such that the function

$$
f(z):=\sum_{n} n^{\ln n} p_{n}(z)
$$

is holomorphic in $B$, and for each hyperplane $\Pi \subset \mathbb{C}^{N}$ and any $p>0$,

$$
\int_{\Pi \cap B}|f(z)|^{p} d m_{\Pi}(z)=\infty
$$

$\left(m_{\Pi}\right.$ is the Lebesgue measure on $\left.\Pi\right)$. It would be interesting to know whether the construction in the present note, given for an arbitrary balanced domain of holomorphy, can be done more explicitly, e.g. as in [10].

\section{References}

[1] J.-P. Ferrier, Spectral Theory and Complex Analysis, North-Holland, 1973.

[2] J. Globevnik and E. L. Stout, Highly noncontinuable functions on convex domains, Bull. Sci. Math. 104 (1980), 417-434.

[3] G. M. Henkin, Integral representation of functions holomorphic in strictly pseudoconvex domains and applications to the $\bar{\partial}$-problem, Math. USSR-Sb. 11 (1970), $273-281$.

[4] P. Jakóbczak, Highly nonintegrable functions in the unit ball, Israel J. Math. 97 (1997), 175-181.

[5] J. Janas, On a theorem of Lebow and Mlak for several commuting operators, Studia Math. 76 (1983), 249-253.

[6] S. G. Krantz, Function Theory of Several Complex Variables, Wiley, 1982.

[7] I. Lieb, Die Cauchy-Riemannschen Differentialgleichungen auf streng pseudokonvexen Gebieten I, Math. Ann. 190 (1970), 6-44.

[8] J. Siciak, Highly noncontinuable functions on polynomially convex sets, Zeszyty Naukowe Uniw. Jagiell. 25 (1985), 95-107.

[9] S. Trapani, Complex retractions and envelopes of holomorphy, Proc. Amer. Math. Soc. 104 (1988), 145-148.

[10] P. Wojtaszczyk, On highly nonintegrable functions and homogeneous polynomials, Ann. Polon. Math. 65 (1997), 245-251. 
[11] A. Zeriahi, Ensembles pluripolaires exceptionnels pour la croissance partielle des fonctions holomorphes, ibid. 50 (1989), 81-91.

Institute of Mathematics

Cracow University of Technology

Warszawska 24

31-155 Kraków, Poland

E-mail: jakobcza@im.uj.edu.pl

Reçu par la Rédaction le 15.12.1997 